\title{
Avaliação dos resultados da artrodese da articulação escapulotorácica no tratamento da escápula alada na distrofia fascioescapulumeral*
}

\author{
Evaluation of scapulothoracic arthrodesis results in the \\ treatment of winged scapula in facioscapulohumeral dystrophy
}

\author{
luciano antonio nassar Pellegrino', Dulce Egydio de Carvalho', \\ Gustavo Barbosa Celia Hinkenickel ${ }^{1}$, Alberto Naoki MiyazakI ${ }^{2}$, Pedro DoneuX-Santos ${ }^{3}$,

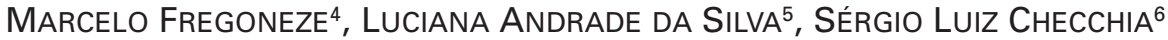

\section{RESUMO}

Objetivo: Avaliar a técnica cirúrgica da artrodese escapulotorácica na distrofia fascioescapulumeral (DFEU), analisando os resultados e as complicações pós-operatórias. Métodos: No período de fevereiro de 1992 a fevereiro de 2006 foram realizadas

* Trabalho realizado no Grupo de Ombro e Cotovelo do Departamento de Ortopedia e Traumatologia da Faculdade de Ciências Médicas da Santa Casa de São Paulo, "Pavilhão Fernandinho Simonsen" (DOT-FCMSCSP).

1. Médico-Residente do Departamento de Ortopedia e Traumatologia da Faculdade de Ciências Médicas da Santa Casa de São Paulo - DOT-FCMSCSP - São Paulo (SP), Brasil.

2. Professor Instrutor; Doutor e Instrutor do Grupo de Ombro e Cotovelo - DOT-FCMSCSP - São Paulo (SP), Brasil.

3. Médico Assistente do Departamento; Instrutor do Grupo de Ombro e Cotovelo - DOT-FCMSCSP - São Paulo (SP), Brasil.

4. Professor Assistente; Instrutor do Grupo de Ombro e Cotovelo DOT-FCMSCSP - São Paulo (SP), Brasil.

5. Médica Assistente do Departamento; Instrutora do Grupo de Ombro e Cotovelo - DOT-FCMSCSP - São Paulo (SP), Brasil.

6. Professor Adjunto; Chefe do Grupo de Ombro e Cotovelo - DOTFCMSCSP - São Paulo (SP), Brasil.

Endereço para correspondência: DOT-FCMSCSP, Rua Dr. Cesário Mota Jr., 112 - 01220-020 - São Paulo (SP), Brasil. Tel./fax: (11) 3222-6866.

E-mail: ombro@ombro.med.br. Site: http://www.ombro.med.br Recebido em 18/7/07. Aprovado para publicação em 24/1/08. Copyright RBO2008 oito artrodeses escapulotorácicas em cinco pacientes no Departamento de Ortopedia e Traumatologia da Faculdade de Ciências Médicas da Santa Casa de São Paulo (DOT-FCM-SCSP). Os critérios para indicação cirúrgica foram: dor, déficit funcional do membro acometido, fadiga muscular e deformidade estética. Na técnica cirúrgica empregada para a artrodese foi realizada a fixação da escápula à parede torácica por meio de amarrilho com fios de poliéster no ${ }^{\circ}$, uma placa metálica estreita e fina, além de colocação de enxerto esponjoso autólogo. Resultados: O seguimento médio dos pacientes foi de 124 meses. Na comparação da amplitude de movimentos pré e pós-operatórios, notou-se melhora na elevação, mantida a rotação lateral, com o UCLA no período pré-operatório variando de 7 a 11 e pós-operatório de 29 a 33. Dentre as complicações, encontraram-se dois casos de pneumotórax, um caso de soltura do material de síntese e um caso de ausência de consolidação óssea. Comentário: Obtida consolidação da artrodese em seis casos, além da melhora da dor e elevação. Dois casos foram reoperados, sendo um devido à quebra do material e o outro, à não consolidação. Todos evoluíram para consolidação.

Descritores - Distrofias musculares; Distrofia muscular fascioescapulumeral; Escápula; Artrodese; Estudos de avaliação 


\section{ABSTRACT}

Objective: To evaluate the surgical scapulothoracic arthrodesis technique in facioscapulohumeral dystrophy (FSHD) by analyzing post-op results and complications. Methods: from February 1992 to February 2006, eight scapulothoracic arthrodesis procedures were performed in five patients at the Orthopedics and Traumatology Department of the Medical Sciences School at the Santa Casa Hospital of São Paulo (DOT-FCM-SCSP). The criteria for surgical indication were pain, functional deficit of the limb involved, muscular fatigue, and esthetic deformity. The surgical technique used for the arthrodesis fixated the scapula to the thoracic wall by tying a narrow, slim plate with No. 5 polyester threads and placing an autologous cancellous bone graft. Results: Mean follow-up of the patients was of 124 months. Comparing the range of movement before and after surgery, the authors observed an improvement in raising, lateral rotation being kept, with the pre-op UCLA ranging from 7 to 11, and the post-op UCLA ranging from 29 to 33. Complications included two cases of pneumothorax; one case of the detachment of synthesis material, and one case of lack of bone fusion. Comment: Arthrodesis fusion was achieved in six cases, besides improvement in pain and raising. Two cases were reoperated on, one of them due to breakage of the material and another one due to lack of fusion. All cases evolved on to fusion.

Keywords - Muscular dystrophies; Facioscapulohumeral muscular dystrophy; Scapula; Arthrodesis; Evaluation studies

\section{INTRODUÇÃO}

A distrofia fascioescapulumeral (DFEU) é um quadro de início insidioso, progressivo e caracteriza-se pela degeneração da musculatura da cintura escapular, causando fraqueza desta. Clinicamente, o paciente apresenta escápula alada, além da visível atrofia muscu$\operatorname{lar}^{(1)}$. A doença pode afetar a musculatura da face e, nos quadros mais graves, os membros inferiores, com progressiva diminuição da força e com acometimen- to, geralmente, assimétrico ${ }^{(1)}$. $\mathrm{O}$ envolvimento facial pode causar ptose palpebral, queda da rima labial e alteração da mímica ${ }^{(1)}$. Nos membros inferiores pode levar à deformidade em eqüino do pé e perda progressiva da capacidade de deambulação ${ }^{(1)}$.

Tawil et al citam que Landouzy e Dejerine, em 1884, foram os primeiros a descrever a DFEU como sendo um distúrbio hereditário, autossômico dominante e de penetrância variável, cuja freqüência é de aproximadamente uma para 20 mil pessoas ${ }^{(2)}$. A faixa etária mais acometida pela afecção está entre os 15 e 30 anos. A musculatura mais freqüentemente envolvida compreende o trapézio, serrátil anterior, grande dorsal e rombóides. O músculo deltóide e o manguito rotador são poupados, mantendo sua função ${ }^{(3)}$.

$\mathrm{Na}$ literatura internacional foram descritas algumas técnicas estabilizadoras da escápula junto ao gradil costal, utilizando uma banda do músculo tensor da fascia lata e, recentemente, a artrodese escapulotorácica com enxerto ósseo ${ }^{(3)}$.

Este trabalho tem como objetivo avaliar a técnica cirúrgica da artrodese articular escapulotorácica realizada no DOT-FCM-SCSP, por meio da análise dos resultados e das complicações pós-operatórias em pacientes com DFEU.

\section{MÉTODOS}

No período de março de 1992 a fevereiro de 2006, no DOT-FCM-SCSP foram realizadas oito artrodeses da articulação escapulotorácica em cinco pacientes com DFEU. A média de idade dos pacientes foi de 31 anos (22 a 40 anos) (tabela 1).

A média de acompanhamento dos pacientes no período pós-operatório foi de 124 meses (mínimo de 23 e máximo de 186 meses) (tabela 1).

Os indivíduos afetados referiam dor progressiva no ombro, impossibilidade de realizar atividades habituais como pentear os cabelos, alimentar-se e alcançar objetos acima da cabeça (déficit de elevação do membro superior acometido) (figura 1). No sexo feminino, além da dor, a queixa mais freqüente foi a deformidade estética devida à escápula alada. A indicação de cirurgia baseou-se na presença desses sintomas citados. Os pacientes que manifestavam a distrofia no lado domi- 
TABELA 1

Dados clínicos dos pacientes

\begin{tabular}{|c|c|c|c|c|c|c|c|c|c|c|c|}
\hline Nome & Idade & D & $\begin{array}{c}\mathrm{AF} \\
\text { casos }\end{array}$ & $\begin{array}{c}\text { Evol. } \\
\text { (meses) }\end{array}$ & $\begin{array}{l}\text { Acomp. } \\
\text { (meses) }\end{array}$ & Mob. pré & Mob. pós & Intra-op & Comp. pós-op & UCLApré & UCLApós \\
\hline 1. $A A A$ & 26 & + & 2 & 12 & 163 & $(65,50, T 7)$ & $(140,60, T 5)$ & - & - & 9 & 33 \\
\hline $\begin{array}{l}\text { 2. JFL } \\
\text { JFL }\end{array}$ & $\begin{array}{l}39 \\
40\end{array}$ & $\begin{array}{l}+ \\
-\end{array}$ & 2 & $\begin{array}{l}132 \\
132\end{array}$ & $\begin{array}{r}107 \\
95\end{array}$ & $\begin{array}{l}(90,30, \mathrm{~T} 12) \\
(45,45, \mathrm{~T} 7)\end{array}$ & $\begin{array}{l}(110,40, \text { T9) } \\
(95,40, \text { T9) }\end{array}$ & Pneumotórax & $\begin{array}{l}- \\
\text { soltura do material } \\
\text { reop com sucesso }\end{array}$ & $\begin{array}{r}11 \\
7\end{array}$ & $\begin{array}{l}33 \\
32\end{array}$ \\
\hline $\begin{array}{l}\text { 3. MMM } \\
\text { MMM }\end{array}$ & $\begin{array}{l}29 \\
30\end{array}$ & $\begin{array}{l}+ \\
-\end{array}$ & 4 & $\begin{array}{l}120 \\
156\end{array}$ & $\begin{array}{l}137 \\
104\end{array}$ & $\begin{array}{l}(70,80, T 1) \\
(70,80, T 2)\end{array}$ & $\begin{array}{l}(120,30, \mathrm{~T} 6) \\
(120,30, \mathrm{~T} 7)\end{array}$ & $\begin{array}{l}- \\
-\end{array}$ & $\begin{array}{l}- \\
-\end{array}$ & $\begin{array}{l}10 \\
10\end{array}$ & $\begin{array}{l}32 \\
32\end{array}$ \\
\hline 4. RCD & 38 & + & - & 180 & 23 & $(90,60, T 7)$ & $(90,45$, Sacro $)$ & - & - & 9 & 30 \\
\hline $\begin{array}{r}\text { 5. SB } \\
\text { SB }\end{array}$ & $\begin{array}{l}22 \\
22\end{array}$ & $\begin{array}{l}+ \\
-\end{array}$ & 1 & $\begin{array}{l}120 \\
120\end{array}$ & $\begin{array}{l}186 \\
182\end{array}$ & $\begin{array}{l}(70,20, T 7) \\
(45,40, T 7)\end{array}$ & $\begin{array}{l}(110,80, T 7) \\
(80,60, T 7)\end{array}$ & $\begin{array}{c}- \\
\text { Pneumotórax }\end{array}$ & $\begin{array}{c}- \\
\text { não consolidação } \\
\text { reop com sucesso }\end{array}$ & $\begin{array}{l}8 \\
8\end{array}$ & $\begin{array}{l}32 \\
29\end{array}$ \\
\hline
\end{tabular}

Legenda: $\mathrm{D}$ = dominância; $\mathrm{AF}$ = antecedentes familiares com distrofia; Evol. = evolução da doença pré-operatória; Acomp. = acompanhamento pós-operatório; Mob. pré = mobilidade pré-operatória; Mob. pós = mobilidade pós-operatória; Intra-op = complicações intra-operatórias; Comp. pós-op = complicações pós-operatórias; UCLApré = UCLA pré-operatório; UCLApós = UCLA pós-operatório; reop = reoperado.

Fonte: SAME - Santa Casa de Misericórdia de São Paulo.

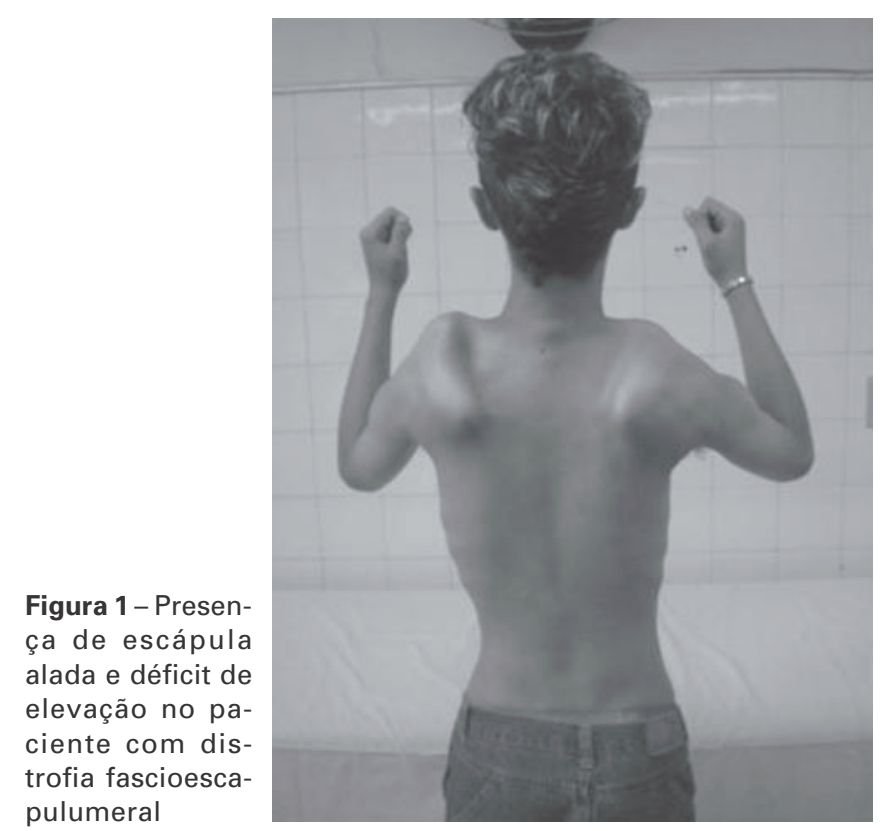

nante (cinco dos oito casos) possuíam grande limitação em suas atividades de vida diária.

Todos os pacientes tinham força muscular normal do deltóide e do manguito rotador.

Quatro dos cinco pacientes tinham história familiar de distrofia fascioescapulumeral (tabela 1).

Antes do procedimento cirúrgico, a média de elevação ativa encontrada no exame físico dos cinco pacientes foi de $68^{\circ}$ (variando de 45 a $90^{\circ}$ ), rotação late- ral ativa de $48^{\circ}$ (variando de 20 a $80^{\circ}$ ) e rotação medial ativa de T6 (variando de T1 a T12) (tabela 1).

Com relação à técnica cirúrgica da artrodese, o paciente é submetido à anestesia geral e colocado em decúbito ventral. O membro a ser operado permanece livre a fim de que a escápula fique em rotação lateral de $20^{\circ}$ a $25^{\circ}$. O correto posicionamento do membro deixando-o totalmente livre é fundamental no momento da estabilização da escápula à caixa torácica.

A incisão é realizada no sentido longitudinal, na borda medial da escápula. O trapézio é desinserido da espinha da escápula; os músculos rombóide maior, rombóide menor e elevador da escápula são dissecados de suas inserções na borda medial da escápula. $\mathrm{Na}$ DFEU, esses músculos estão atrofiados em grau variado.

Em seguida, a superfície anterior da escápula é preparada, ressecando-se subperiostalmente parte dos músculos serrátil anterior e subescapular para permitir maior contato entre o enxerto e a borda medial da escápula, na ocasião da artrodese. São decorticados sete arcos costais na altura da superfície escapular a ser fixada, com o objetivo de manter maior área para fusão. Entretanto, cinco arcos costais são utilizados para artrodese, de forma a minimizar as complicações pulmonares relacionadas ao amarrilho da escápula ao arco costal. Atenção deve ser dada à decorticação dos arcos costais, uma vez que existe íntima relação entre 

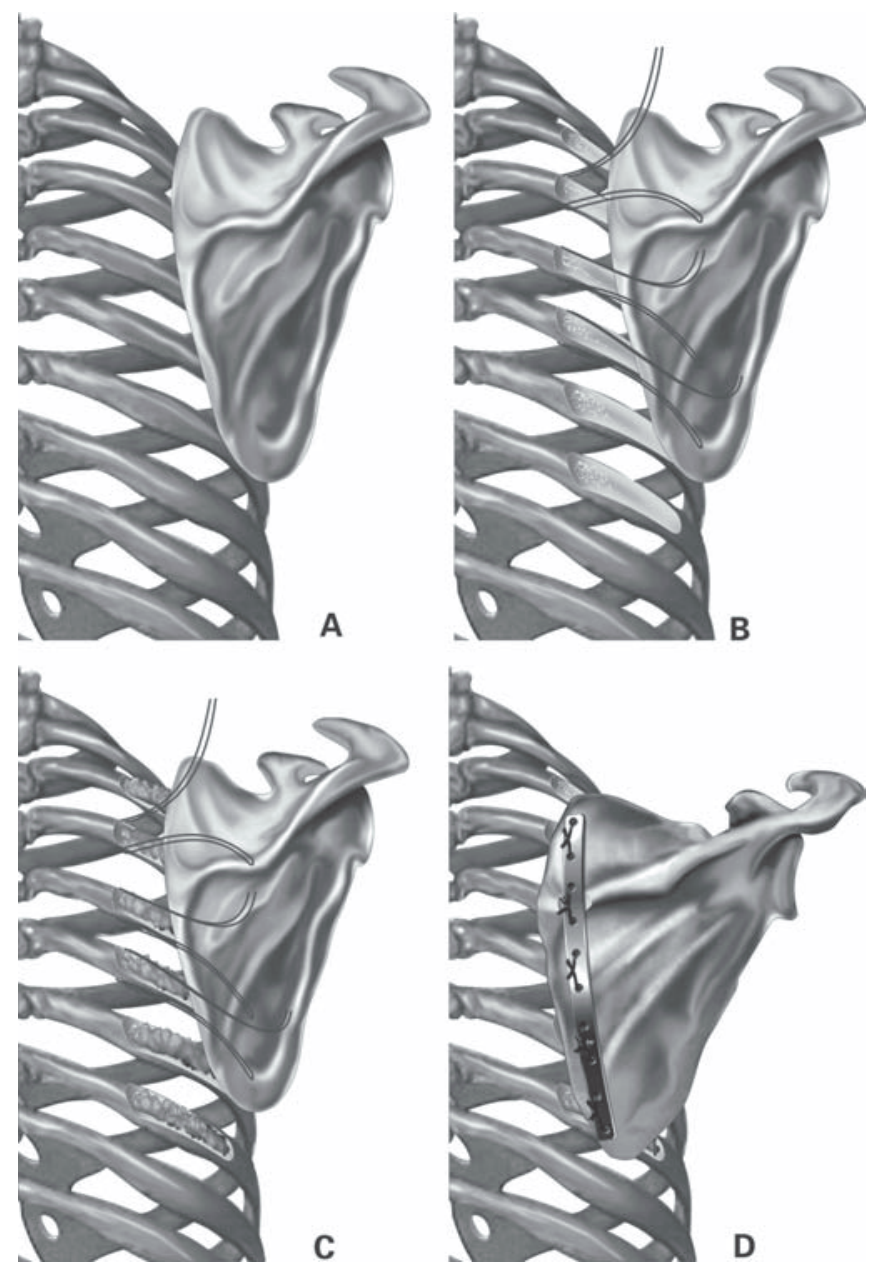

Figura 2 - Ilustração da artrodese escapulotorácica: A) visão posterior do ombro direito; B) passagem do fio subcostal; C) cruentização dos arcos + colocação de enxerto esponjoso; D) posicionamento do material de síntese + fixação.

a pleura parietal e estruturas neurovasculares no trajeto de dissecção. A escápula deve ser reduzida à sua posição anatômica de forma cuidadosa para evitar estiramento do plexo braquial ${ }^{(1)}$.

Enxerto esponjoso é retirado da crista ilíaca. Uma placa especial estreita e fina de cinco a sete furos é posicionada na borda dorsal da escápula para dar suporte ao frágil osso dessa região. São realizados cinco furos na escápula através da placa, correspondendo à altura dos arcos costais a serem fixados. Uma camada de enxerto de osso esponjoso, retirado do ilíaco, é posicionada entre a escápula e os arcos costais decorticados. É necessária cautela durante a passagem dos

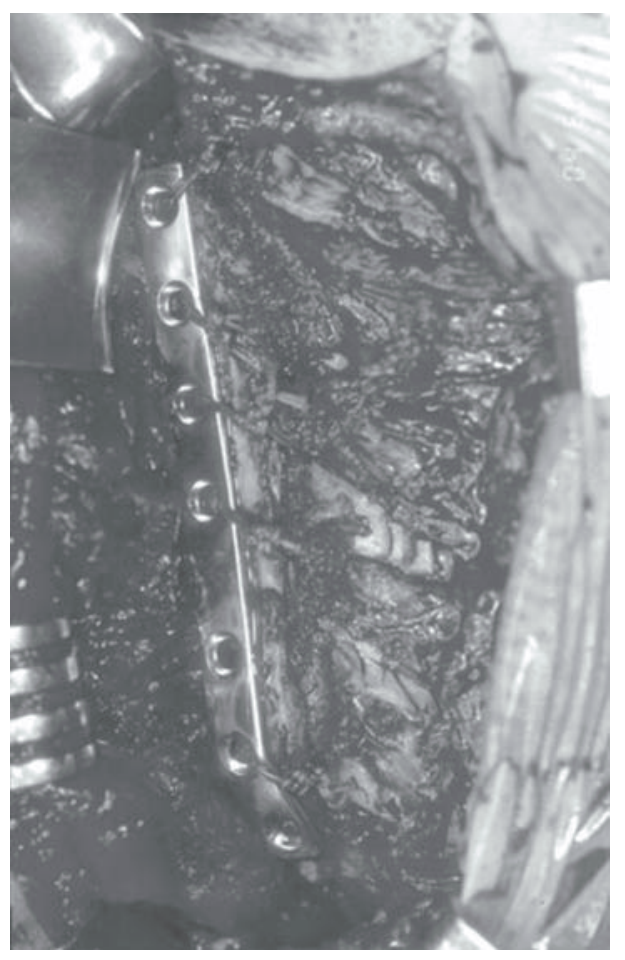

Figura 3 - Foto intra-operatória do posicionamento do material de síntese na escápula direita

fios subcostais, utilizando uma rugina delicada para descolamento subperiostal dos arcos costais, assim evitando a ocorrência de pneumotórax.

Ancora-se a placa à escápula, fixando-a à caixa torácica com o auxílio de dois fios inabsorvíveis (poliéster no 5) para cada arco costal. O amarrilho com os fios é realizado de forma seqüencial, enquanto um auxiliar mantém a escápula em $20^{\circ}$ a $25^{\circ}$ de abdução (figuras 2 e 3 ).

Nesse momento, o campo operatório é preenchido com soro fisiológico a $0,9 \%$ e, ao solicitar-se ao anestesista que expanda o pulmão do paciente, a presença de bolhas de ar diagnostica um pneumotórax, que deve ser drenado ao final do ato cirúrgico ortopédico, quando presente.

No período pós-operatório o membro superior acometido permanece imobilizado com tipóia de lona por aproximadamente 12 semanas, tempo estimado da consolidação da artrodese. A mobilização ativa do cotovelo, punho e mão é estimulada precocemente. Comprovadas as consolidações clínica e radiográfica, inicia-se a reabilitação do ombro. 

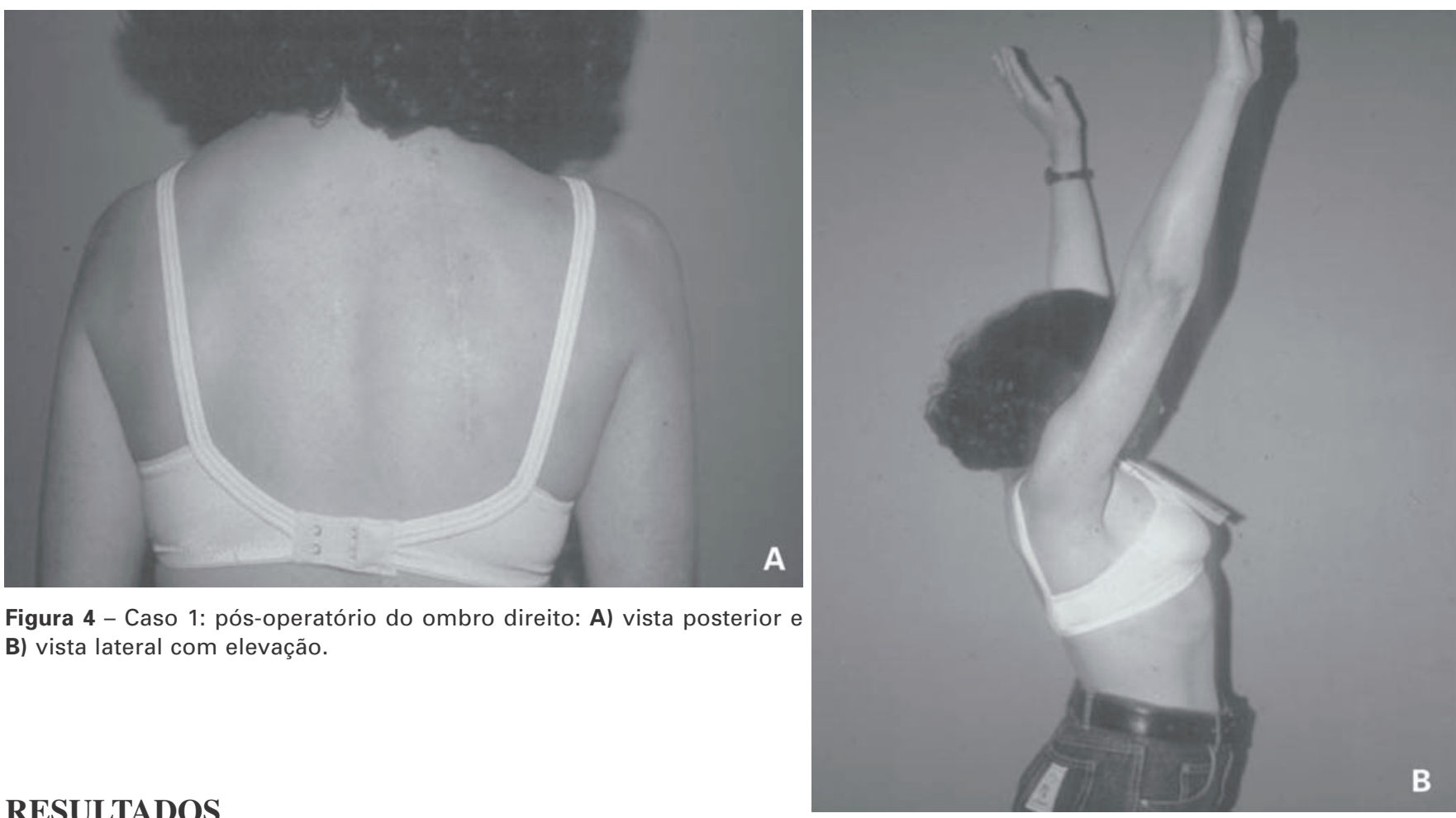

Figura 4 - Caso 1: pós-operatório do ombro direito: A) vista posterior e B) vista lateral com elevação.

\section{RESULTADOS}

Para a avaliação dos resultados do procedimento foi utilizado o protocolo UCLA: avaliação clínica e radiográfica da artrodese. Em relação à amplitude de movimento, todos os pacientes obtiveram ganho da elevação do ombro acometido (média de $30^{\circ}$ ). Os pacientes possuíam média de elevação de $68^{\circ}$ (variando de $45^{\circ}$ a $90^{\circ}$ ) previamente à cirurgia e, após o procedimento, a média foi de $108^{\circ}$ (variando de $80^{\circ}$ a $140^{\circ}$ ) (figuras $3 \mathrm{e}$ 4). Em relação à rotação lateral, não houve melhora da função (média pré-operatória: $50^{\circ}$; pós-operatória: $\left.48^{\circ}\right)$. Quatro de oito casos operados (50\%) evoluíram com diminuição da rotação medial (nível médio préoperatório: T6; pós-operatório: T8).

Todos os pacientes deste trabalho receberam pontuação pré-operatória segundo o critério UCLA inferior a 21 pontos (ruim), sendo a média de 9 (7 a 11) e, após a cirurgia, todos apresentaram pontuação entre 28 e 33 pontos (bom), com média de 31,5 (29 a 33) (tabela 1).

Os cinco pacientes referiram retorno às suas atividades profissionais prévias e melhora da função de realizar as de vida diária. Todos os pacientes relataram dor no período pré-operatório e referiram melho- ra da mesma após a artrodese da articulação escapulotorácica. Não houve queixas relacionadas à cicatriz cirúrgica.

Todos relataram melhora da aparência estética. Essa melhora está associada à ausência de escápula alada. No exame físico pós-operatório dos casos de fixação bilateral (três casos) notou-se simetria dos ombros.

Dois pacientes tiveram pneumotórax no momento da manipulação dos arcos costais. Nos dois casos, o diagnóstico foi feito durante o ato cirúrgico e confirmado radiograficamente; os dois casos foram submetidos à drenagem imediata do tórax, com resolução do quadro em 48 horas.

Consolidaram seis das oito artrodeses. Um paciente apresentou ausência de consolidação à esquerda, sendo realizado novo procedimento, que evoluiu com sucesso. Em outro paciente, em que a cirurgia foi bilateral, houve soltura do material de síntese do lado esquerdo após um mês. O paciente foi reoperado com a mesma técnica, obtendo-se consolidação após três meses. 


\section{DISCUSSÃO}

A DFEU é um distúrbio muscular extremamente incapacitante para o indivíduo após 10 a 20 anos do início dos sintomas; a doença é progressiva e a função dos membros acometidos fica muito limitada ${ }^{(3)}$.

A elevação e a abdução do membro superior estão prejudicadas na DFEU, decorrente da fraqueza muscular paravertebral ${ }^{(4)}$. Quando o deltóide e os músculos do manguito rotador contraem, a fim de movimentar o ombro, os estabilizadores da escápula, insuficientes, não a mantêm junto ao gradil costal, ocorrendo a translação posterior e rotação medial da mesma, sendo esse o mecanismo da escápula alada ${ }^{(4)}$.

Rose et al realizaram estudos randomizados sobre a eficácia do tratamento farmacológico para a DFEU, com o uso de beta-2-agonistas e creatina. Nenhum deles mostrou melhora nos sintomas, tampouco impediu a progressão da doença ${ }^{(5)}$. Acredita-se que o melhor tratamento atualmente é cirúrgico, mas a grande dificuldade é determinar qual o melhor momento para sua realização.

Métodos variados de estabilização da articulação escapulotorácica foram utilizados no passado sem que houvesse resultado satisfatório ${ }^{(1-4,6-7)}$. As transferências musculares não mostraram bons resultados, pois não permitiam boa estabilização da escápula, uma vez que a musculatura era insuficiente ou distrófica ${ }^{(1-4)}$. A fixação unicamente com parafusos e enxerto ósseo, segundo Copeland et al em uma série de 11 casos, resultou em fratura por estresse dos arcos costais em dois pacientes, possivelmente decorrente de parafusos salientes na caixa torácica ${ }^{(4)}$. A técnica utilizada com o auxílio de banda de fascia lata não promovia estabilização permanente devido ao estiramento com subseqüente afrouxamento da fixação ${ }^{(8)}$.

Copeland et al citam que Rinaldi fixou a escápula ao tórax com uma banda do músculo tensor da fascia lata, envolvendo os processos espinhosos de T2 a T6 através de um orifício confeccionado na espinha da escápula ${ }^{(3)}$. Essa foi uma modificação da cirurgia de Dewar e Harris, que combinava a transferência do músculo peitoral maior fixando-o na escápula por meio de alças de fáscia muscular. Os resultados obtidos foram insatisfatórios devido ao desgaste e afrouxamento da fáscia ${ }^{(3,9)}$.
Howard publicou resultados de 12 artrodeses escapulotorácicas em oito pacientes utilizando fixação com parafusos e enxerto ósseo da região proximal da tíbia ${ }^{(3,10)}$. Apesar dos bons resultados, alguns pacientes referiram dor pleural ${ }^{(3)}$. Letournel et al introduziram a técnica de fusão escapulotorácica com o auxílio de placas, parafusos e amarrilho com fios de aço ${ }^{(8)}$.

No Brasil, Ferreira Filho et al utilizaram a técnica de fusão escapulotorácica com fio de poliéster no 5 e enxerto esponjoso da crista ilíaca, obtendo consolidação de cinco artrodeses em sete ombros operados ${ }^{(11)}$. Um desses pacientes recusou-se a realizar outro procedimento, porém o outro foi reoperado com sucesso. A idade dos pacientes variou de 17 a 26 anos. A técnica cirúrgica preconizada era semelhante à proposta no estudo em questão, fixando a escápula ao quarto, quinto, sexto e sétimo arcos costais, utilizando sutura com fio de poliéster no 5 e enxerto esponjoso do osso ilíaco. Não houve complicações pulmonares ${ }^{(11)}$.

Segundo Krishnan et al, em um estudo de artrodese escapulotorácica em 24 ombros de pacientes portadores de diversas doenças, $46 \%$ dos pacientes apresentaram complicações pulmonares utilizando a técnica de fixação com placa e amarrilho com fios de aço. Em nosso estudo, os dois pacientes que apresentaram pneumotórax foram drenados no centro cirúrgico com sua resolução completa após 48 horas ${ }^{(12)}$. Realizamos de rotina uma radiografia de tórax no pós-operatório imediato de todos os pacientes.

De acordo com Mackenzie et al, deve-se observar o correto posicionamento da escápula durante a artrodese para evitar compressões neurovasculares, devidas à tração ou compressão do plexo braquial. Em nossa série não tivemos complicações neurológicas ou alteração de perfusão ${ }^{(13)}$.

A técnica cirúrgica realizada neste estudo é semelhante àquela preconizada por Diab et al ${ }^{(1)}$, porém com algumas modificações: os rombóides são suturados à borda medial da escápula sobre o implante e um dreno aspirativo é usado. Segundo Diab et al, dos 11 casos submetidos à artrodese, dois evoluíram com resultado regular devido à fadiga muscular do músculo deltóide; não evidenciada na nossa amostra no período de acompanhamento pós-operatório(1) ${ }^{(1)}$ 
No presente trabalho, houve um caso de soltura do material após um mês, o paciente foi reoperado e no intra-operatório foi observado que os fios estavam partidos. Não sabemos se houve falha na técnica cirúrgica empregada nesse paciente ou imprudência no seguimento das orientações pelo mesmo no período pós-operatório. A realização do amarrilho entre a escápula e os arcos costais é executada com fio de poliéster no 5 em vez do fio de aço; acreditamos que a incidência de pneumotórax é reduzida durante a passagem do fio no arco costal e permite fixação estável tanto quanto o fio de aço.

Os resultados de amplitude de movimento demonstrados após a cirurgia foram concordantes com os relatados na literatura. Em alguns trabalhos os parâmetros mais utilizados foram flexão e abdução do ombro ${ }^{(1,3,8,14-16)}$; já outros consideraram a elevação, rotação lateral e medial como parâmetros para avaliação da mobilidade ${ }^{(4,12)}$.

Neste estudo utilizamos como parâmetro a elevação e as rotações do ombro para avaliação. Foi obtida grande melhora na elevação ativa dos ombros operados, média de $68^{\circ}$ inicialmente, a $101^{\circ}$, no pós-operatório. Já a rotação medial se manteve em dois casos e piorou em quatro casos. Provavelmente, esse problema é inerente à artrodese da articulação escapulotorácica.

O sucesso da artrodese escapulotorácica foi evidenciado pelo critério do UCLA, avaliado antes e após a cirurgia. Todos os pacientes receberam pontuação superior a 28 pontos, correspondendo a sete resultados bons $(87,5 \%)$ e um excelente $(12,5 \%)$. Nenhum resultado insatisfatório foi registrado (tabela 1).

Em dois casos houve complicações: ruptura dos fios e conseqüente não consolidação; foram reoperados com sucesso, o que não comprometeu o resultado final, estando os pacientes satisfeitos.

\section{COMENTÁRIOS}

A avaliação dos resultados da artrodese escapulotorácica demonstrou a melhora: do movimento de elevação do membro superior operado, da dor e da função dos ombros submetidos a ela, segundo protocolo da UCLA aplicado no pré e pós-operatório.

Foi obtida a consolidação em todos os casos, apesar de que em dois casos houve a necessidade de reoperação.

\section{REFERÊNCIAS}

1. Diab M, Darras BT, Shapiro F. Scapulothoracic fusion for facioscapulohumeral muscular dystrophy. J Bone Joint Surg Am. 2005;87(10):2267-75.

2. Tawil R, Van Der Maarel SM. Facioscapulohumeral muscular dystrophy. Muscle Nerve. 2006;34(1):1-15.

3. Copeland SA, Levy O, Warner GC, Dodenhoff RM. The shoulder in patients with muscular dystrophy. Clin Orthop Relat Res. 1999;(368):80-91.

4. Copeland SA, Howard RC. Thoracoscapular fusion for facioscapulohumeral dystrophy. J Bone Joint Surg Br. 1978; 60(4):547-51.

5. Rose MR, Tawil R. Drug treatment for facioscapulohumeral muscular dystrophy. Cochrane Database Syst Rev. 2004;(2): CD002276.

6. Jakab E, Gledhill RB. Simplified technique for scapulocostal fusion in facioscapulohumeral dystrophy. J Pediatr Orthop. 1993;13(6):749-51.

7. Ketenjian AY. Scapulocostal stabilization for scapular winging in facioscapulohumeral muscular dystrophy. J Bone Joint Surg Am. 1978;60(4):476-80.

8. Letournel E, Fardeau M, Lytle JO, Serrault M, Gosselin RA. Scapulothoracic arthrodesis for patients who have fascioscapulohumeral muscular dystrophy. J Bone Joint Surg Am. 1990;72(1):78-84.

9. Dewar FP, Harris RI. Restoration of function of the shoulder following paralysis of the trapezius by fascial sling fixation and transplantation of the levator scapule. Ann Surg. 1950;132(6): 1111-5.

10. Howard RC. Thoraco-scapular arthrodesis. J Bone Joint Surg Br. 1961;43:175. Abstract.

11. Ferreira AA Filho, Zoppi A Filho, Bolliger R Neto, Greve JMDA, Ferreira AA Neto, Carazzato, JG. A fusão escápulo-torácica na distrofia fáscio-escápulo-umeral. Rev Bras Ortop. 1992;27(9): 642-4.

12. Krishnan SG, Hawkins RJ, Michelotti JD, Litchfield R, Willis RB, Kim YK. Scapulothoracic arthrodesis: indications, technique and results. Clin Orthop Relat Res. 2005;(435):12633.

13. Mackenzie WG, Riddle EC, Earley JL, Sawatzky BJ. A neurovascular complication after scapulothoracic arthrodesis. Clin Orthop Relat Res. 2003;(408):157-61.

14. Berne D, Laude F, Laporte C, Fardeau M, Saillant G. Scapulothoracic arthrodesis in facioscapulohumeral muscular dystrophy. Clin Orthop Relat Res. 2003;(409):106-13.

15. Andrews CT, Taylor TC, Patterson VH. Scapulothoracic arthrodesis for patients with facioscapulohumeral muscular dystrophy. Neuromuscul Disord. 1998;9(8):580-4.

16. Bunch WH, Siegel IM. Scapulothoracic arthrodesis in facioscapulohumeral muscular dystrophy. Review of seventeen procedures with three to twenty-one-year follow-up. J Bone Joint Surg Am. 1993;75(3):372-6. 